\title{
Differentiating biochemical from clinical heparin resistance in COVID-19
}

\author{
Ton Lisman $^{1}$ [D $\cdot$ Jecko Thachil ${ }^{2}$
}

Published online: 3 September 2020

(c) Springer Science+Business Media, LLC, part of Springer Nature 2020

With interest, we read the recent paper by White and coworkers in which heparin resistance in patients with COVID19 patients on the intensive care unit was described [1]. Accumulating evidence shows that patients with COVID-19 are at high risk for thrombotic events, even those that receive normal to increased doses of thromboprophylaxis with low molecular weight heparin or unfractionated heparin [2,3]. The observation that thromboprophylaxis doesn't prevent thrombotic events in a proportion of patients suggests that thromboprophylactic heparins insufficiently downregulate coagulation. White et al. demonstrate a failure to achieve heparin target levels as measured by APTT or anti-Xa assays. In addition, spiking of patient plasma with LMWH led to a lower than expected anti-Xa levels in plasma. These observations led the authors to conclude COVID-19 patients are heparin resistant.

We wish to comment on the concept of heparin resistance, and offer an alternative explanation for the findings presented by White et al. Heparin resistance would ideally be defined as a decreased capacity of unfractionated or lowmolecular weight heparin to downregulate coagulation in a thrombotic environment. In a heparin-responsive patient, adequate downregulation of coagulation leads to a hemostatic state that, depending on the heparin dose, prevents or treats thrombotic events. In a heparin-resistant patient, inadequate downregulation of coagulation would lead to a sustained risk of thrombotic events in patients receiving thromboprophylaxis, or a lack of response in patients with an acute event. However, the biochemical response to heparins

Ton Lisman

j.a.lisman@umcg.nl

1 Surgical Research Laboratory and Section of Hepatobiliary Surgery and Liver Transplantation, Department of Surgery, University Medical Center Groningen, BA33, Hanzeplein 1, 9713 GZ Groningen, The Netherlands

2 Department of Haematology, Manchester University Hospitals, Oxford road, Manchester, UK is poorly defined in clinical practice. Functional tests to assess downregulation of coagulation by heparins are not yet available for clinical use. Such tests include thrombin generation tests that are able to assess functional efficacy of heparins by assessing thrombin generation in samples taken from patients prior to and while on heparin therapy. The proportional decrease of thrombin generation would be an indicator for the anticoagulant efficacy of heparin, and inadequate downregulation of coagulation would then qualify as 'biochemical heparin resistance'.

In clinical practice, however, heparin resistance is defined by unusually high heparin doses required to reach target APTT or anti-Xa levels in combination with a failure of heparins to prevent or treat thrombotic events. Heparin resistance is common, with a $22 \%$ incidence in patients undergoing cardiac surgery with cardiopulmonary bypass [4]. In patients undergoing cardiopulmonary bypass, $65 \%$ of cases with heparin resistance are associated with acquired antithrombin deficiency. In addition, elevated levels of heparin-binding proteins may contribute to heparin resistance [5]. In COVID-19, acquired antithrombin deficiency is uncommon, but does occur in individual patients even in a small proportion of patients that are not acutely ill ([6], and Lisman unpublished results). High levels of heparin binding proteins, which are associated with an acute phase reaction are likely common in COVID-19 patients.

We propose that a 'clinical heparin resistance' is not necessarily caused by 'biochemical heparin resistance'. An anti-Xa test is not a functional coagulation test, but rather provides a measure of heparin concentration in a plasma sample. As shown by White et al., and well known from other studies [7, 8], certain anti-Xa tests underestimate the true heparin concentration in samples with decreased antithrombin levels. It is therefore plausible that the decreased anti-Xa levels in the spiking experiments in plasma from COVID-19 patients performed by White et al. do not indicate heparin resistance, but rather an analytical problem. Heparin concentrations in patients with decreased 
antithrombin levels can be accurately assessed using anti-Xa assays to which antithrombin has been added to the reagent mixture. Similarly, heparin resistance based on failure to achieve target APTT levels may be caused by elevated factor VIII levels [9], and again this heparin resistance likely does not reflect a true biochemical heparin resistance, but a failure to adequately quantify heparin levels.

Spuriously low anti-Xa of APTT ratio levels in plasma from COVID-19 patients therefore do not necessarily mean that downregulation of coagulation by heparins in these samples is inadequate. Recent studies have shown a profound hypercoagulable state in plasma from patients with COVID-19, with a decrease in thrombin generating potential with increasing heparin concentrations in the sample $[6,10]$. These studies suggest that the tremendously elevated baseline hemostatic potential, rather than a heparin resistance explains the high thrombotic risk of COVID-19 patients, even in the presence of prophylactic or elevated doses of heparins. In other words, heparin in COVID-19 patients may be able to downregulate coagulation to the same extent as in a non-COVID-19 patient, with a similar proportional decrease in thrombin generation. However, due to the profound hypercoagulable state at baseline, on-heparin hemostatic potential is still high, explaining the risk for thrombotic events.

We have previously shown that in patients with liver disease, who often have acquired antithrombin deficiency, anti-Xa and APTT tests are not suitable to estimate heparin concentrations in the sample [11]. While anti-Xa tests profoundly underestimate heparin levels, thrombin generation tests show that heparins are effective in downregulating coagulation [12].

Thus, failure to achieve APTT or anti-Xa target levels in a COVID-19 patient are likely not indicating a true biochemical heparin resistance, but rather a failure to adequately quantify heparin levels in plasma. Anti-Xa tests to which antithrombin is added to the reagent mixture would be preferred when monitoring heparins in COVID-19 patients. Well-designed studies using research-type assays such as thrombin generation tests are required to define whether COVID-19 patients are truly resistant to the anticoagulant action of heparins, or whether the high baseline hemostatic potential, perhaps in combination with profound triggers of activation of coagulation such as damaged epithelium in the lung or hypoxia [13], indeed explains thrombotic risk in these patients, even in the presence of prophylactic or higher doses of heparin.

\section{References}

1. White D, MacDonald S, Bull T, Hayman M, Monteverde-Robb $\mathrm{R}$, de Sapsford D et al (2020) Heparin resistance in COVID-19 patients in the intensive care unit. J Thromb Thrombolysis. https ://doi.org/10.1007/s11239-020-02145-0

2. Levi M, Hunt BJ (2020) Thrombosis and coagulopathy in COVID19: an illustrated review. Res Pract Thromb Haemost 4:744-751

3. Iba T, Levy JH, Levi M, Thachil J (2020) Coagulopathy in COVID-19. J Thromb Haemost. https://doi.org/10.1111/jth.14975

4. Spiess BD (2008) Treating heparin resistance with antithrombin or fresh frozen plasma. Ann Thorac Surg 85(6):2153-2160

5. Young E, Podor TJ, Venner T, Hirsh J (1997) Induction of the acute-phase reaction increases heparin-binding proteins in plasma. Arterioscler Thromb Vasc Biol 17(8):1568-1574

6. Blasi A, von Meijenfeldt FA, Adelmeijer J, Calvo A, Ibañez C, Perdomo J, Carlos Reverter J, Lisman T (2020) In vitro hypercoagulability and ongoing in vivo activation of coagulation and fibrinolysis in COVID-19 patients on anticoagulation. J Thromb Haemost. https://doi.org/10.1111/jth.15043

7. Croles FN, Lukens MV, Mulder R, de Maat MPM, Mulder AB, Meijer K (2019) Monitoring of heparins in antithrombin-deficient patients. Thromb Res 175:8-12

8. Vera-Aguilera J, Yousef H, Beltran-Melgarejo D, Teng TH, Jan R, Mok M, Vera-Aguilera C, Moreno-Aguilera E (2016) Clinical scenarios for discordant Anti-Xa. Adv Hematol 2016:4054806

9. Levine MN, Hirsh J, Gent M, Turpie AG, Cruickshank M, Weitz J, Anderson D, Johnson M (1994) A randomized trial comparing activated thromboplastin time with heparin assay in patients with acute venous thromboembolism requiring large daily doses of heparin. Arch Intern Med 154(1):49-56

10. Nougier C, Benoit R, Simon M, Desmurs-Clavel H, Marcotte G, Argaud L et al (2020) Hypofibrinolytic state and high thrombin generation may play a major role in sars-cov2 associated thrombosis. J Thromb Haemost. https://doi.org/10.1111/jth.15016

11. Potze W, Arshad F, Adelmeijer J, Blokzijl H, van den Berg AP, Porte RJ et al (2013) Routine coagulation assays underestimate levels of antithrombin-dependent drugs but not of direct anticoagulant drugs in plasma from patients with cirrhosis. Br J Haematol 163:666-673

12. Potze W, Arshad F, Adelmeijer J, Blokzijl H, Van Den Berg AP, Meijers JCM et al (2014) Differential in vitro inhibition of thrombin generation by anticoagulant drugs in plasma from patients with cirrhosis. PLoS ONE. https://doi.org/10.1371/journ al.pone. 0088390

13. Thachil J (2020) Hypoxia — an overlooked trigger for thrombosis in COVID-19 and other critically ill patients. J. Thromb. Haemost. https://doi.org/10.1111/jth.15029

Publisher's Note Springer Nature remains neutral with regard to jurisdictional claims in published maps and institutional affiliations. 